\title{
Weak-lensing halo numbers and dark-matter profiles
}

\author{
M. Bartelmann ${ }^{1}$, L. J. King ${ }^{1,2}$, and P. Schneider ${ }^{2}$ \\ 1 Max-Planck-Institut für Astrophysik, PO Box 1317, 85741 Garching, Germany \\ 2 Institut für Astronomie und Extraterrestrische Forschung, Universität Bonn, Auf dem Hügel 71, \\ 53121 Bonn, Germany
}

Received 28 March 2001 / Accepted 6 August 2001

\begin{abstract}
Integral measures of weak gravitational lensing by dark-matter haloes, like the aperture mass, are sensitive to different physical halo properties dependent on the halo mass density profile. For isothermal profiles, the relation between aperture mass and virial mass is steeper than for haloes with the universal NFW profile. Consequently, the halo mass range probed by the aperture mass is much wider for NFW than for isothermal haloes. We use recent modifications to the Press-Schechter mass function in CDM models normalised to the local abundance of rich clusters, to predict the properties of the halo sample expected to be accessible with the aperture mass technique. While $\sim 10$ haloes should be detected per square degree if the haloes have NFW profiles, their number density is lower by approximately an order of magnitude if they have isothermal profiles. These results depend only very mildly on the cosmological background model. We conclude that counts of haloes with a significant weak-lensing signal are a powerful discriminator between different dark-matter profiles.
\end{abstract}

Key words. galaxies: clusters: general - gravitational lensing

\section{Introduction}

One of the manifestations of gravitational lensing is the coherent distortion of the images of faint background galaxies near foreground matter concentrations due to their tidal gravitational field. This effect is now well established for lensing by large-scale structure (e.g. Van Waerbeke et al. 2000; Bacon et al. 2000; Kaiser et al. 2000; Wittman et al. 2000; Maoli et al. 2001; Van Waerbeke et al. 2001), in which case it is called "cosmic shear", and for lensing by galaxy clusters and groups. Following pioneering work by Kaiser \& Squires (1993), detailed two-dimensional mass maps of galaxy clusters were constructed (e.g. Fischer \& Tyson 1997; Clowe et al. 2000; Hoekstra et al. 2000) and the weaker effect of galaxy groups were stacked to quantify their mean properties (Hoekstra et al. 2001). See for example Mellier (1999) and Bartelmann \& Schneider (2001) for recent reviews on weak lensing.

Being sensitive only to projected total mass, weak gravitational lensing also allows the detection of sufficiently massive haloes only by the effects of their mass, regardless of their composition, physical state and relation to luminous material (Erben et al. 2000). Cumulative measures for their gravitational tidal field, or shear, were

Send offprint requests to: M. Bartelmann,

e-mail: msb@mpa-garching.mpg.de proposed, among which the so-called aperture mass (Kaiser et al. 1994; Schneider 1996) is most directly related to observations. The aperture mass is a suitably weighted integral of the surface mass density inside a circular aperture, which is equivalent to a differently weighted integral over the net tangential shear within the aperture. The aperture mass is therefore a direct observable.

It was recently shown in a different context (Bartelmann 2001) that it depends on the density profile of a halo as to which physical halo property the aperture mass actually measures. While the aperture mass is $\propto M^{2 / 3}$ for singular isothermal profiles, it is $\propto M^{1 / 3}$ for the universal profile proposed by Navarro et al. (1997, hereafter NFW), with $M$ being the virial halo mass.

In this paper, we study the consequences of this difference in detail. The basic idea is this. Given a fixed sensitivity limit of aperture mass measurements, the flatter dependence of aperture mass on virial mass for NFW haloes compared to isothermal haloes implies that aperture mass measurements can cover a broader physical mass range if the halo population has NFW rather than isothermal profiles. The physical masses accessible to aperture mass measurements correspond to rich groups and clusters, whose mass function is steep, turning over from power-law to exponential decrease with increasing mass. Even a small change in the accessible mass range can thus give rise to 
substantial changes in the numbers of haloes expected to be detectable with the aperture mass method. Conversely, this implies that number counts of haloes with significant aperture mass can sensitively constrain the halo density profiles, without the need to obtain detailed density profiles of individual haloes.

The plan of the paper is as follows. We summarise the aperture mass and its relevant properties in Sect. 2, and description of the halo population in Sect. 3. Results are shown in Sect. 4 and Sect. 5 contains a discussion and our conclusions.

\section{Aperture mass and halo profiles}

\subsection{Aperture mass}

The aperture mass, suggested in Schneider (1996) to quantify the weak-lensing effects of dark-matter haloes, is an integral over the lensing convergence $\kappa$ within a circular aperture of angular radius $\theta$, weighted by a function $U(\vartheta)$ which vanishes outside the aperture,

$M_{\mathrm{ap}}(\theta)=\int \mathrm{d}^{2} \vartheta \kappa(\boldsymbol{\vartheta}) U(|\boldsymbol{\vartheta}|)$

The prime advantage of $M_{\mathrm{ap}}$ is that it can directly be determined from the measured tidal distortions of background-galaxy images in the chosen aperture, provided $U(\vartheta)$ is compensated, i.e.

$2 \pi \int_{0}^{\theta} \mathrm{d} \vartheta \vartheta U(\vartheta)=0$.

A broad class of weighting functions satisfies this condition. In Schneider et al. (1998) the simple form was suggested,

$U(\vartheta)=\frac{9}{\pi \theta^{2}}\left[1-\left(\frac{\vartheta}{\theta}\right)^{2}\right]\left[\frac{1}{3}-\left(\frac{\vartheta}{\theta}\right)^{2}\right]$

within the aperture, and $U(\vartheta)=0$ outside.

The aperture mass depends on source redshift because the convergence $\kappa$ does. Since this is a linear dependence, and $M_{\text {ap }}$ depends linearly on $\kappa$, a realistic source redshift distribution like

$p\left(z_{\mathrm{s}}\right)=\frac{\beta}{z_{0}^{3} \Gamma(3 / \beta)} \exp \left[-\left(\frac{z}{z_{0}}\right)^{\beta}\right]$

can easily be taken into account by averaging $M_{\text {ap }}$ over $p\left(z_{\mathrm{s}}\right)$. We choose $\beta=1.5$ and $z_{0}=1.0$ here and assume from now on that source-redshift averaged aperture masses are being used. For simplicity, we denote them by $M_{\text {ap }}$ as before, as there is no risk of confusion.

Schneider (1996) also calculated the dispersion of $M_{\mathrm{ap}}$ due to the finite number of randomly distributed background galaxies and their intrinsic ellipticities. Assuming typical values for the number density $n_{\mathrm{g}}$ of suitably bright background galaxies and for the dispersion $\sigma_{\epsilon}$ of their intrinsic ellipticities, it was found that

$$
\begin{aligned}
\sigma_{\mathrm{M}}(\theta)= & 0.016\left(\frac{n_{\mathrm{g}}}{30 \operatorname{arcmin}^{2}}\right)^{-1 / 2}\left(\frac{\sigma_{\epsilon}}{0.2}\right) \\
& \times\left(\frac{\theta}{1 \operatorname{arcmin}}\right)^{-1} .
\end{aligned}
$$

It can be shown by means of the Cauchy-Schwarz inequality that the signal-to-noise ratio for a given halo is maximised if the weighting function $U(\vartheta)$ is chosen such that it follows the halo density profile. With the latter unknown, this cannot generally be achieved, but we shall show later that the optimisation of weighting functions is of minor importance.

The aperture size $\theta$ should be chosen large enough to encompass a substantial number of background galaxies and to significantly cover the extent of dark-matter haloes, and small enough in comparison with typical field sizes and typical separations between neighbouring haloes. Aperture sizes of a few arc minutes seem appropriate; to be specific, we choose $\theta=3^{\prime}$ in the following unless stated otherwise (see also Kruse \& Schneider 1999).

\subsection{Mass profiles}

Let us now investigate the effect on the aperture mass if the density profile of a halo of fixed physical mass $M$ is changed. We choose two alternative density profiles, the singular isothermal sphere and the profile suggested by NFW. We assume that halo masses are virial masses, i.e. masses confined by the virial radius $r_{200}$,

$r_{200}=\left(\frac{G M}{100 H^{2}(z)}\right)^{1 / 3}$

where $H(z)$ is the Hubble function at redshift $z$. The virial radius is defined such that the mean density within $r_{200}$ is 200 times the critical density. Temperature $T$ and virial mass $M$ are assumed to be related by

$k T=(k T)_{15} M_{15}^{2 / 3}(1+z)\left(\frac{\Omega_{0}}{\Omega(z)}\right)^{1 / 3}\left(\frac{\Delta_{\mathrm{c}}}{178}\right)^{1 / 3}$

(e.g. Eke et al. 1996; Navarro et al. 1995), where $M_{15}=$ $M / 10^{15} M_{\odot}$ and $(k T)_{15}=6.03 \mathrm{keV}$ is the temperature of a cluster with $M_{15}=1$ (Mathiesen \& Evrard 2001). The density parameter at redshift $z$ is $\Omega(z)$, and $\Delta_{\mathrm{c}}$ is the mean overdensity of a virialised sphere (e.g. Łokas \& Hoffman 2001).

\subsubsection{Singular isothermal spheres}

A singular isothermal sphere has the density profile

$\rho(r)=\frac{\sigma_{\mathrm{v}}^{2}}{2 \pi G r^{2}}$ 
The (radially constant) velocity dispersion along the lineof-sight, $\sigma_{\mathrm{v}}$, is empirically related to the cluster temperature by

$\sigma_{\mathrm{v}}=10^{2.57 \pm 0.08}\left(\frac{k T}{\mathrm{keV}}\right)^{0.56 \pm 0.09} \mathrm{~km} \mathrm{~s}^{-1}$

(Wu et al. 1998), which is in turn related to the cluster mass by (7). The velocity-temperature relation has a large scatter and is compatible with the theoretical expectation $\sigma_{\mathrm{v}} \propto T^{1 / 2}$. It is important for the following that the velocity dispersion scales with mass approximately as

$\sigma_{\mathrm{v}} \propto M^{1 / 3}$.

Projecting the density profile (8) along the line-of-sight and scaling the resulting surface mass density $\Sigma$ with its critical value for lensing,

$\Sigma_{\mathrm{cr}}=\frac{c^{2}}{4 \pi G} \frac{D_{\mathrm{s}}}{D_{\mathrm{d}} D_{\mathrm{ds}}}$,

we arrive at the lensing convergence

$\kappa(\vartheta)=\frac{\theta_{\mathrm{E}}}{2 \vartheta}$,

where $\theta_{\mathrm{E}}$ is the angular Einstein radius,

$\theta_{\mathrm{E}}=4 \pi \frac{\sigma_{\mathrm{v}}^{2}}{c^{2}} \frac{D_{\mathrm{ds}}}{D_{\mathrm{s}}}$

Here and in (11), $D_{\mathrm{d}, \mathrm{s}, \mathrm{ds}}$ are the angular diameter distances between observer and lens, observer and source, and lens and source, respectively.

Inserting (12) and (3) into (1), we find the aperture mass for the singular isothermal sphere,

$M_{\mathrm{ap}}^{(\mathrm{SIS})}(\theta)=\frac{4}{5} \frac{\theta_{\mathrm{E}}}{\theta}$.

Since the Einstein radius scales with $\sigma_{\mathrm{v}}^{2}$, we see from (10) that

$M_{\mathrm{ap}}^{(\mathrm{SIS})}(\theta) \propto M^{2 / 3}$.

\subsubsection{NFW haloes}

NFW used numerical $N$-body simulations to show that relaxed haloes assume the universal density profile

$\rho(r)=\frac{\rho_{\text {crit }} \delta_{\mathrm{c}}}{\left(r / r_{\mathrm{s}}\right)\left(1+r / r_{\mathrm{s}}\right)^{2}}$,

where $\rho_{\text {crit }}$ is the critical density and $\delta_{\mathrm{c}}$ is a characteristic overdensity. The characteristic radial scale $r_{\mathrm{s}}$ is related to the virial radius through $r_{\mathrm{s}}=r_{200} / c$, where $c$ is the concentration parameter. Bartelmann (1996) showed that the NFW profile has the lensing convergence

$\kappa(x)=\frac{2 \kappa_{\mathrm{s}}}{x^{2}-1}\left(1-\frac{2}{\sqrt{1-x^{2}}} \operatorname{arctanh} \sqrt{\frac{1-x}{1+x}}\right)$,

with the convergence scale

$\kappa_{\mathrm{s}} \equiv \frac{\rho_{\mathrm{crit}} \delta_{\mathrm{c}} r_{\mathrm{s}}}{\Sigma_{\mathrm{cr}}}$ and $x \equiv r / r_{\mathrm{s}}$. NFW described how the parameters $\delta_{\mathrm{c}}$ and $r_{\mathrm{s}}$ are related to the virial mass $M$ of the halo. Hence, despite the two formal parameters in the profile (16), it is entirely determined once the halo mass is fixed. The statistics of dark-matter haloes described by the NFW density profile has also been investigated by Kruse \& Schneider (1999).

Unfortunately, there is no closed expression for the aperture mass of an NFW profile. Numerically, however, it turns out to be fairly shallow, approaching a constant for small apertures $\theta \rightarrow 0$. It is therefore appropriate to expand the aperture mass into a series in

$t \equiv \frac{\theta}{\theta_{\mathrm{s}}}$

were $\theta_{\mathrm{s}}$ is the angular scale radius, $\theta_{\mathrm{s}}=r_{\mathrm{s}} / D_{\mathrm{d}}$. We find

$$
\begin{aligned}
M_{\mathrm{ap}}^{(\mathrm{NFW})}(t) \approx & \kappa_{\mathrm{S}}\left[1+t^{2}\left(\frac{19}{32}+\frac{3}{4} \ln \frac{t}{2}\right)\right. \\
+ & \left.t^{4}\left(\frac{77}{160}+\frac{3}{4} \ln \frac{t}{2}\right)\right],
\end{aligned}
$$

which shows that $M_{\mathrm{ap}}^{(\mathrm{NFW})} \rightarrow \kappa_{\mathrm{S}}$ in the limit of small apertures. For haloes massive enough to produce a significant weak-lensing signal, $\rho_{\text {crit }} \delta_{\mathrm{c}}$ varies only very little with mass. Therefore, $M_{\mathrm{ap}}^{(\mathrm{NFW})}$ essentially measures the scale radius $r_{\mathrm{s}}$ or, equivalently,

$M_{\mathrm{ap}}^{(\mathrm{NFW})} \propto M^{1 / 3}$

cf. Eq. (6).

Equations (21) and (15) show that the aperture mass measures different physical quantities depending on the density profile of a given halo. Although the scaling with virial mass is not wildly different between singular isothermal spheres and NFW haloes, the effect on weak-lensing observations of a halo population can be substantial, as we shall show below. For a given virial halo mass, the virial radius is determined by (6). Irrespective of the density profile, the halo mass within $r_{200}$ is therefore fixed. For the isothermal profile, the mass grows linearly with radius to reach $M$ at $r_{200}$, while it grows more rapidly for $r \lesssim r_{\mathrm{s}}$ and less rapidly beyond $r_{\mathrm{s}}$ in case of an NFW density profile. The mass enclosed by $r$ in an NFW halo thus exceeds that in a singular isothermal halo for all $r \leq r_{200}$. An NFW halo concentrates more mass in a given radius $r \leq r_{200}$, and therefore produces a stronger weak lensing effect for a given aperture size if $r \gtrsim r_{\mathrm{s}}$. Although the previous scaling (21) was derived in the limit of small apertures, $t \ll 1$, the preceding argument shows that the main result will continue to hold also for larger apertures, namely that an NFW halo needs less mass to produce the same weak-lensing signal as a singular isothermal halo.

\subsubsection{Weighting functions}

As mentioned before, $M_{\text {ap }}$ can be optimised by choosing the weight function $U(\vartheta)$ such that it follows the halo density profile (Schneider 1996). Of course, this cannot completely be achieved because of the requirement (2) that 


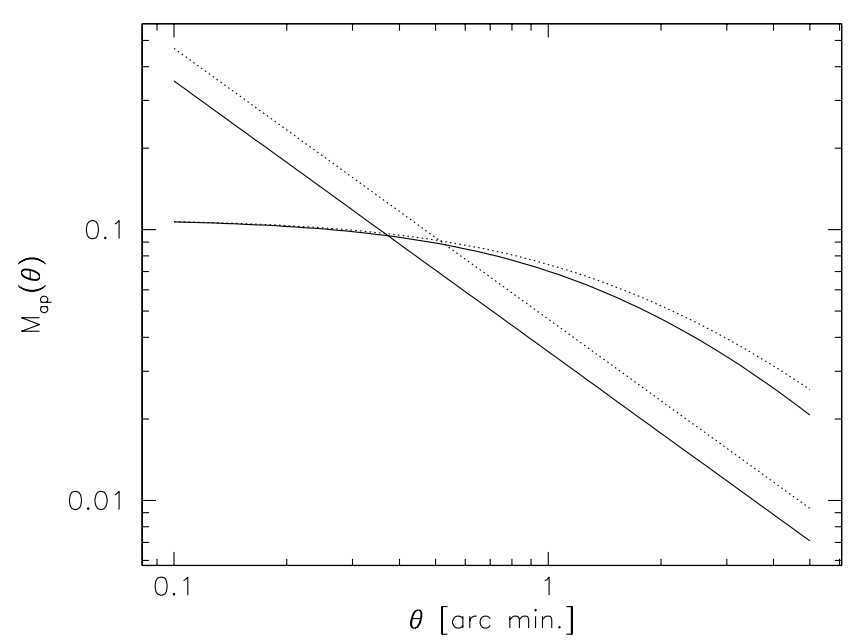

Fig. 1. Aperture mass profiles $M_{\mathrm{ap}}(\theta)$ for a singular isothermal sphere (steeper curves) and an NFW halo (flatter curves) with mass $10^{14} M_{\odot} / h$ at redshift 0.2 . In this figure, the source redshift is 1.0. While $M_{\text {ap }}$ falls like $\theta^{-1}$ for the SIS, it flattens for small $\theta$ for the NFW case. The solid curves were obtained with the weighting function (3), while (22) was used to obtain the dotted curves. The optimised weighting function (22) improves $M_{\text {ap }}$ by $\sim 30 \%$ for the singular isothermal sphere, and less for the NFW profile.

$U(\vartheta)$ be compensated. It is, however, possible to choose the weighting function such that it approximately follows the slope of the halo density profile within most of the aperture. For singular isothermal spheres, such an approximation could be

$U(\vartheta) \propto \frac{1}{\sqrt{\vartheta^{2}+\vartheta_{\mathrm{c}}^{2}}}-C$,

where $\vartheta_{\text {c }}$ removes the central singularity, $C$ must be chosen to satisfy (2) and the normalisation is the same as for (3). For the NFW profile, an optimised weighting function is more difficult to construct because the physical scale $r_{\mathrm{s}}$ changes its angular size with halo distance, so that the weighting function would have to vary with distance. Near $r_{\mathrm{s}}$, where the NFW profile gently steepens from $r^{-1}$ to $r^{-3}$, the profile is approximately isothermal, so some improvement from using (22) instead of (3) is also expected for the NFW profile. Figure 1 shows the aperture mass as a function of aperture size for singular isothermal spheres and NFW haloes, using the weighting functions (3) and (22).

The figure summarises the results obtained in this subsection. While $M_{\mathrm{ap}}(\theta)$ falls $\propto \theta^{-1}$ for the singular isothermal sphere, it flattens for $\theta \rightarrow 0$ for the NFW profile. The weighting function (22), which is adapted to the isothermal profile, improves $M_{\mathrm{ap}}(\theta)$ quite appreciably for the singular isothermal sphere, and less so for the NFW profile. Since the noise $(5)$ is proportional to $\theta^{-1}$, the $\mathrm{S} / \mathrm{N}$ of $M_{\mathrm{ap}}(\theta)$ is constant with $\theta$ for the singular isothermal sphere, and is maximised for the NFW profile at a filter scale of a few arc minutes, where its slope is isothermal.

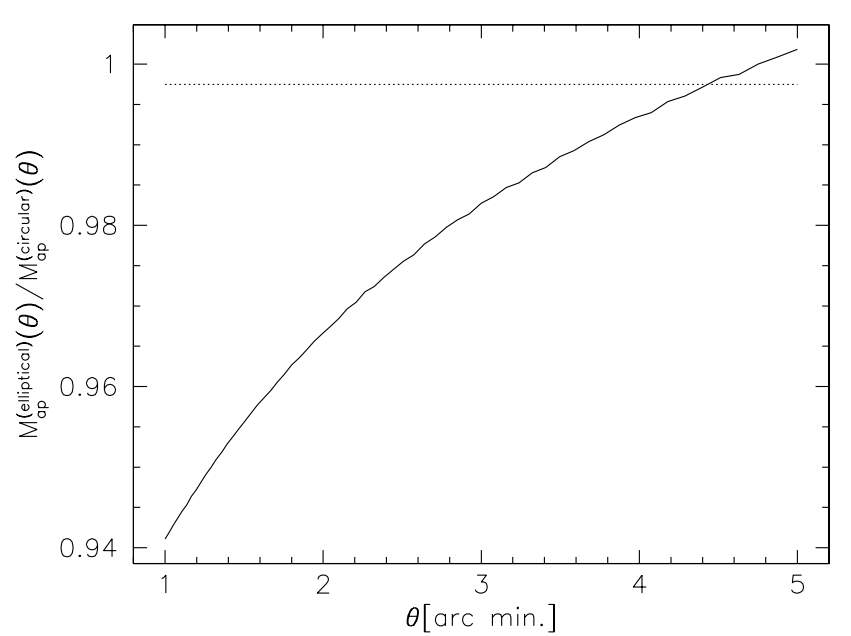

Fig. 2. Influence of halo ellipticity on the aperture mass. The solid curve shows the ratio of the aperture masses for an elliptically distorted NFW halo and one that is circularly symmetric. The assumed ellipticity is $e=0.2$. At an aperture size of $\theta=3^{\prime}$, the ratio deviates from unity by less than 2 per cent. The dotted curve shows the same ratio for singular isothermal profiles, which is independent of aperture size. Here, the deviation from unity is 0.25 per cent.

\subsection{Halo substructure and asymmetry}

Since massive haloes are cosmologically young objects, they frequently show substructure or deviations from axial symmetry. One may wonder whether the relations derived above between aperture mass and virial mass cease to hold if the halo symmetry is perturbed.

For a specific example, let us distort the projected NFW profile (17) such that lines of constant surface density become elliptical, while keeping the azimuthallyaveraged density profile unchanged. This is achieved by multiplying $\kappa(x)$ with $(1-e)^{1 / 2}$ and replacing the radial distance $x$ by

$\xi=\left[x_{1}^{2}+(1-e)^{2} x_{2}^{2}\right]^{1 / 2}$,

where $0 \leq e<1$ is the ellipticity parameter. To first order in $e$, this changes the NFW convergence to

$\tilde{\kappa}(\xi)=\kappa(x)-e\left\{\frac{\kappa(x)}{2}+\frac{x_{2}^{2}}{1-x^{2}}\left[3 \kappa(x)-\frac{2}{x^{2}}\right]\right\}$.

The solid line in Fig. 2 shows the ratio between the aperture masses of the elliptically distorted and the circularly symmetric NFW profiles, $\tilde{\kappa}(\xi)$ and $\kappa(x)$, for $e=0.2$.

The influence of halo ellipticity can also be quantified by comparing $M_{\mathrm{ap}}^{(\mathrm{SIE})}$ for the singular isothermal ellipsoid (Kormann et al. 1994) with $M_{\mathrm{ap}}^{\text {(SIS) }}$. The convergence of the SIE is

$\kappa(\vartheta, \phi)=\frac{\theta_{\mathrm{E}}}{2 \vartheta} \frac{\sqrt{1-e}}{\sqrt{\cos ^{2} \phi+(1-e)^{2} \sin ^{2} \phi}}$, 
where $\phi$ is the position angle with respect to its major axis and $\theta_{\mathrm{E}}$ is the equivalent circular Einstein radius. Note that this reduces to the SIS when $e=0$. We find the closed form

$\frac{M_{\mathrm{ap}}^{(\mathrm{SIE})}}{M_{\mathrm{ap}}^{(\mathrm{SIS})}}=\frac{2 \sqrt{1-e}}{\pi} K\left(\sqrt{1-(1-e)^{2}}\right)$,

where $K(m)$ is the complete elliptic integral of the first kind, following the notation in Gradshteyn \& Ryzhik (1965). There is no dependence upon the particular form of filter function adopted, or upon its scale. The ratio (26) deviates from unity by less than 3 per cent if $e \leq 0.5$, and by less than 11 per cent if $e \leq 0.75$. A Taylor expansion for (26), to second order in $e$ about $e=0$ reads

$\frac{M_{\mathrm{ap}}^{(\mathrm{SIE})}}{M_{\mathrm{ap}}^{(\mathrm{SIS})}}=1-\frac{e^{2}}{16}+\mathcal{O}(e)^{3}$.

For $e=0.2$, this deviates from unity by 0.25 per cent, indicated by the dotted line in Fig. 2 .

Now consider how substructure changes the measured aperture mass, and how the aperture mass obtained for a "realistic" cluster deviates from $M_{\mathrm{ap}}^{(\mathrm{NFW})}$. Intuitively, the presence of substructure in a halo should not influence the aperture mass derived from $\kappa$ provided that the total halo mass remains fixed. The most accurate description of substructure is obtained from high resolution $N$-body simulations, and we use a scaled cluster from Springel (1999); a preliminary consideration of $M_{\text {ap }}$ applied to such simulations was presented in King et al. (2000). For consistency with the simulations we adopt $h=0.7, \Omega_{0}=0.3$ and $\Omega_{\Lambda}=0.7$. The value of $M_{\mathrm{ap}}^{(\mathrm{SIM})}$ obtained for this simulated cluster is the same as that of its azimuthally averaged counterpart, within numerical accuracy.

Figure 3 shows the convergence of the simulated cluster, and of the best-fit NFW model $\left(c=4.9, r_{200}=\right.$ $1.1 \mathrm{Mpc}$ ) over the range plotted. Towards smaller radii, the simulation profile is steeper than NFW, resulting in the best-fit NFW profile having a larger concentration parameter when the inner fitting radius is decreased. The differences between the aperture masses $M_{\mathrm{ap}}^{(\mathrm{SIM})}$ calculated directly from the cluster simulation and $M_{\mathrm{ap}}^{(\mathrm{NFW})}$ for the best-fit NFW model are also negligible for aperture sizes of interest. In other words, the presence of substructure at a level consistent with $N$-body simulations does not significantly change the relationships between aperture mass and virial mass.

\section{Halo population}

An approximate description for the mass function of haloes was given by Press \& Schechter (1976). In terms of mass $M$ and redshift $z$, their mass function can be written as

$$
\begin{aligned}
n_{\mathrm{PS}}(M, z)= & \frac{\bar{\rho}}{\sqrt{2 \pi} D_{+}(z) M^{2}}\left(1+\frac{n}{3}\right)\left(\frac{M}{M_{*}}\right)^{(n+3) / 6} \\
& \times \exp \left[-\frac{1}{2 D_{+}^{2}(z)}\left(\frac{M}{M_{*}}\right)^{(n+3) / 3}\right],
\end{aligned}
$$

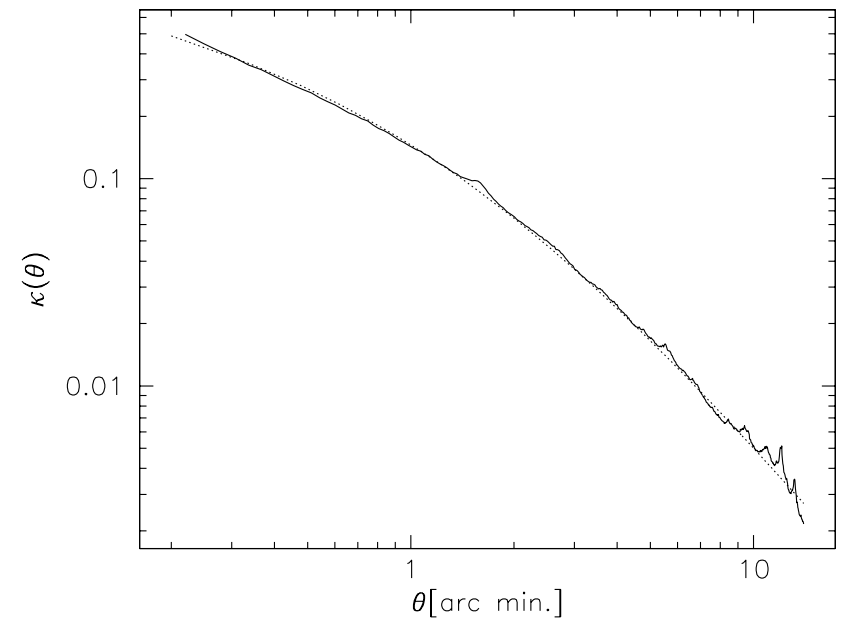

Fig. 3. The true convergence profile of the simulated cluster with substructure (solid line) and the best-fit NFW convergence (dotted line).

where $M_{*}$ and $\bar{\rho}$ are the nonlinear mass today and the mean background density at the present epoch, and $D_{+}(z)$ is the linear growth factor of density perturbations, normalised to unity today, $D_{+}(0)=1$. Finally, $n$ is the effective exponent of the dark-matter power spectrum at the cluster scale, $n \approx-1$.

Sheth \& Tormen (1999) recently modified the mass function (28), and Sheth et al. (2001) introduced ellipsoidal rather than spherical collapse. Jenkins et al. (2001) derived the mass function of dark-matter haloes from numerical simulations and found a fitting formula very close to Sheth \& Tormen's, but with lower amplitude at the high-mass end. Although these different mass functions are very similar over a wide range of masses, their different behaviour at high mass can lead to noticeable changes in our results. We use the mass function by Jenkins et al. for the results shown below.

For rich groups and clusters, the halo mass function is steep in mass. Therefore, even a moderate change in the mass range considered can lead to substantial changes in the total halo number. We saw above that the aperture mass effectively measures different fractional powers of the halo mass depending on the density profile. As the halo mass decreases, $M_{\text {ap }}$ decreases faster for singular isothermal spheres than for haloes with NFW profile. The halo mass range probed by the aperture mass will therefore be narrower if the halo population consists of singular isothermal spheres than if it consists of NFW haloes. Considering the steepness of the halo mass function, we expect to see substantially more haloes with significant aperture mass if the halo population is characterised by the NFW density profile compared to the singular isothermal profile. We will now quantify this expectation. 


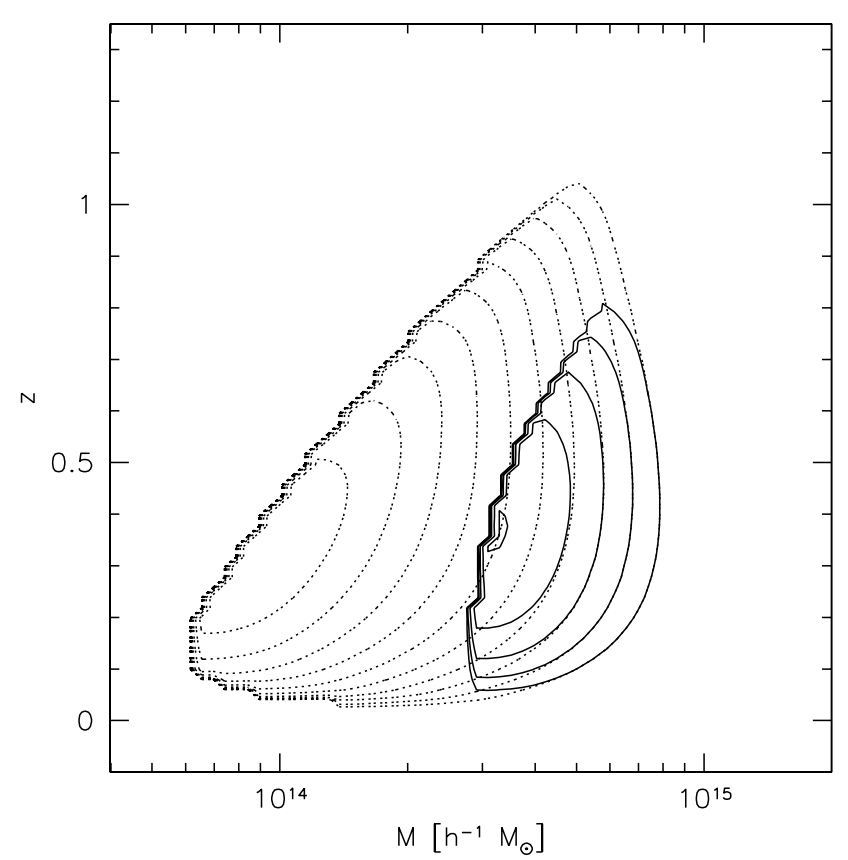

Fig. 4. Number density of haloes $\mathrm{d}^{2} N / \mathrm{d} M \mathrm{~d} z$ in the $M-z$ plane, integrated over the sky, which produce a significant weak-lensing signal. Solid contours: singular isothermal spheres, dashed contours: haloes with NFW density profile. Evidently, the distribution of gravitationally lensing haloes in mass and redshift changes substantially with the density profile. The contour levels range from $10^{-10.5}$ to $10^{-7} h M_{\odot}^{-1}$ and are spaced by 0.25 dex. The cosmology is $\Lambda \mathrm{CDM}\left(\Omega_{0}=0.3\right.$, $\left.\Omega_{\Lambda}=0.7, h=0.7\right)$.

\section{Results}

We describe significant weak gravitational lensing by a halo in terms of the signal-to-noise ratio,

$\mathcal{S}(\theta)=\frac{M_{\mathrm{ap}}(\theta)}{\sigma_{M}(\theta)}$

with $\sigma_{M}(\theta)$ given by (5). To be specific, we require $\mathcal{S} \geq$ $\mathcal{S}_{\text {min }}$ for a significant measurement of the aperture mass, and choose $\mathcal{S}_{\min }=5$ in the following.

In the absence of noise, there would be a one-to-one relation between aperture mass and physical mass, so that a sharp detection cut-off on $M_{\text {ap }}$ would map onto a sharp threshold in mass. However, since the aperture-mass dispersion (5) is finite, some haloes with mass below the threshold have higher, and some haloes with mass above the threshold have lower aperture masses than the cut-off. With the halo mass function falling steeply, on average more low-mass haloes are gained in the sample than highmass haloes lost. We must therefore take this bias into account, which we do by convolving the sharp boundary in mass with a Gaussian of width

$\Delta M=\left(\frac{\partial \mathcal{S}}{\partial M}\right)^{-1}$

Needless to say, $\Delta M$ depends on halo mass and redshift.
Figure 4 shows contours of the number density in the mass-redshift plane of haloes with a significant aperture mass, integrated over the sky. The underlying cosmological model is spatially flat and has low density, $\Omega_{0}=0.3$, $\Omega_{\Lambda}=0.7, h=0.7$, and the dark-matter power spectrum is normalised such that the local abundance of rich clusters is reproduced. The solid contours are for singular isothermal spheres, the dotted contours for NFW haloes. Obviously, the mass range in which NFW haloes can produce a significant weak-lensing signal is much wider than for singular isothermal spheres.

High-redshift lenses come close to, or fall within, the background source population. The number density of lensed background sources is therefore rapidly reduced as the lenses approach $z \sim 1$. We include this effect in our calculations using the redshift distribution (4) for the background sources. At low redshifts, the lensing signal of dark-matter haloes comes close to the background caused by cosmic shear. We ignore this source of noise because it has no impact on our conclusions, as the haloes at lowest redshift contribute only negligibly to the total halo population detectable through weak lensing.

While Fig. 4 qualitatively illustrates the effect expected, the two panels of Fig. 5 show the distribution of weak-lensing haloes in redshift and mass, respectively. Three pairs of curves are shown in each figure for three representative cosmological models, all of which are normalised to reproduce the local cluster abundance. The models are $\Lambda \mathrm{CDM}\left(\Omega_{0}=0.3, \Omega_{\Lambda}=0.7, h=0.7\right)$, OCDM $\left(\Omega_{0}=0.3, \Omega_{\Lambda}=0.0, h=0.7\right)$, and $\operatorname{SCDM}\left(\Omega_{0}=1.0\right.$, $\left.\Omega_{\Lambda}=0.0, h=0.5\right)$. Quite independent of the cosmological model, the peak amplitudes of the curves for singular isothermal spheres are approximately one order of magnitude lower than for NFW haloes.

Figure 6 shows the expected number on the sky of haloes with a significant aperture mass, in dependence on the density parameter $\Omega_{0}$. Two pairs of curves are shown. The upper pair refers to NFW haloes, the lower one to singular isothermal spheres. In each pair, the solid curve was calculated for open universes, $\Omega_{\Lambda}=0$, and the dashed curve for spatially flat universes, $\Omega_{\Lambda}=1-\Omega_{0}$. Note that the Hubble constant is not varied here, $h=0.7$.

On the whole, the expected number of weak-lensing haloes is higher by about an order of magnitude than if the haloes have an NFW rather than a singular isothermal density profile. The dependence on cosmology is very mild, in particular for the NFW haloes, and its trend seems counter-intuitive. Haloes tend to form earlier in low- than in high-density universes, hence one should expect the number of weak-lensing haloes to increase with decreasing $\Omega_{0}$. Contrary to that expectation, the halo number tends to increase slightly with increasing $\Omega_{0}$.

The reason for this trend is the fact that the overdensity inside virialised haloes grows with increasing $\Omega_{0}$. This is most easily seen in the spherical collapse model, in which the virial overdensity for critical-density universes is $\Delta_{\mathrm{c}}=18 \pi^{2} \approx 178$ independent of redshift, but lower by a factor of $\sim 2$ for $\Omega_{0}=0.3$ at low redshifts. Consequently, 

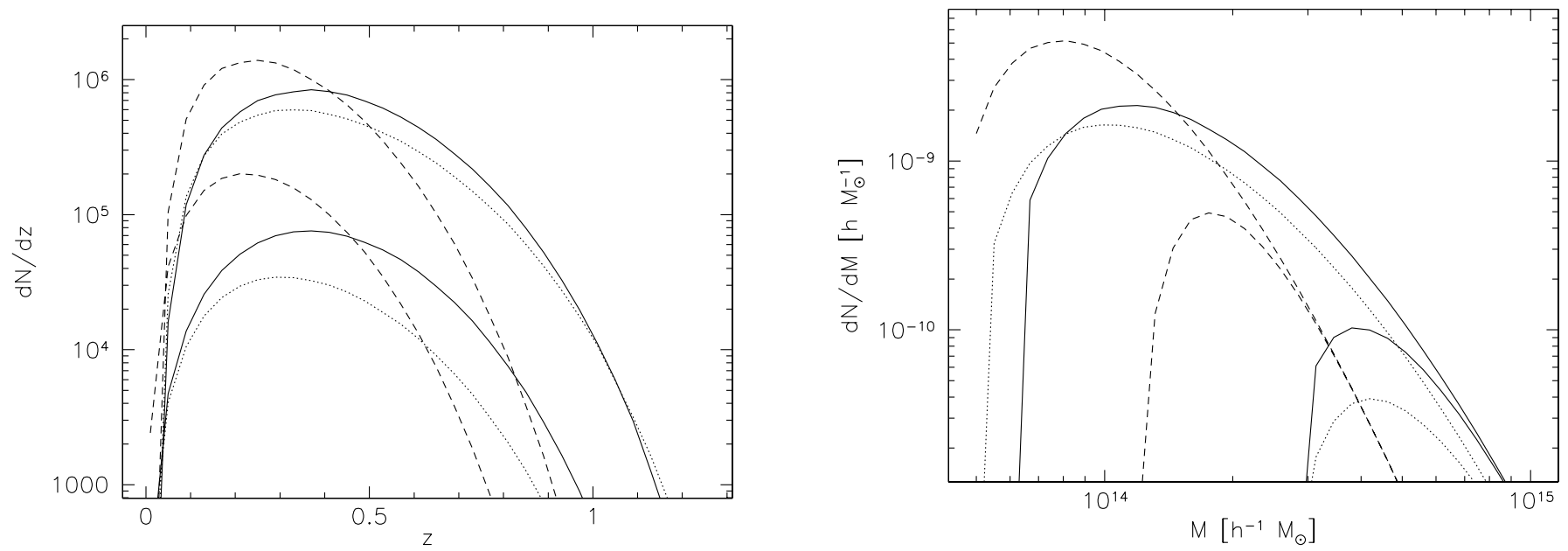

Fig. 5. Distribution of gravitationally lensing haloes in redshift (left panel) and mass (right panel) for three different cosmological models and two different density profiles. Solid curves: $\Lambda$ CDM, dotted curve: OCDM, dashed curve: SCDM. In both panels, the three curves with the lower amplitude refer to SIS haloes, those with the higher amplitude to NFW haloes. While the redshift range is fairly insensitive to the density profile, the mass range reaches to substantially lower mass for a halo population with NFW density profile compared to a population with singular isothermal profile.

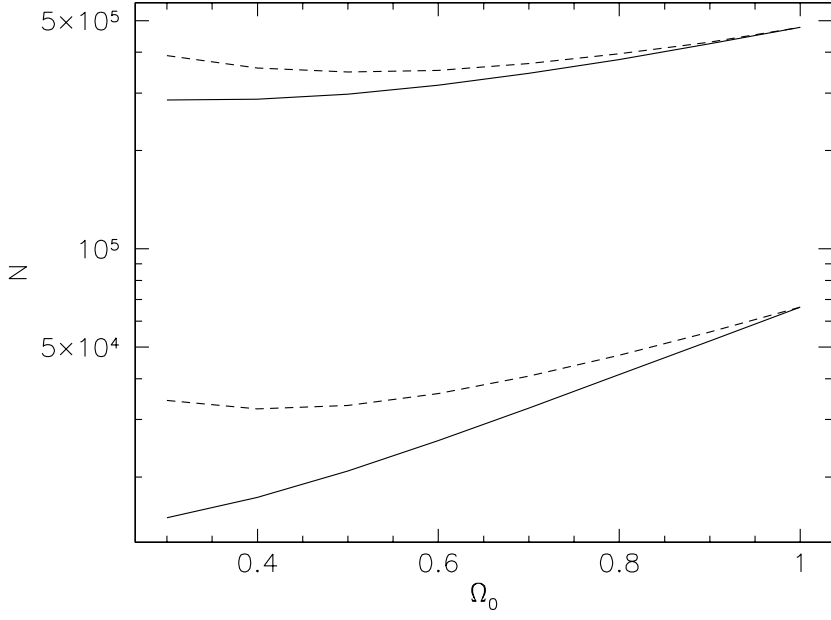

Fig. 6. Total number of gravitationally lensing haloes on the sky as a function of the cosmic density parameter $\Omega_{0}$. Solid curves: $\Omega_{\Lambda}=0$, dashed curves: $\Omega_{\Lambda}=1-\Omega_{0}$. The lower pair of curves refers to SIS haloes, the upper to NFW haloes.

haloes of fixed mass have higher $M_{\text {ap }}$ in high-density universes, or, conversely, haloes that produce a given minimum $M_{\text {ap }}$ can have lower mass in high-density universes. We have seen before that small changes in the mass range probed lead to large changes in the halo numbers because the mass function is so steep. Hence, the higher compactness of haloes in high-density universes leads to an increase in the number of visible haloes that overcompensates for the more rapid decrease in halo number with increasing redshift. Figure 7 further illustrates this point.

\section{Discussion and conclusions}

We studied in this paper how the number of dark-matter haloes with a significant weak-lensing signal depends on

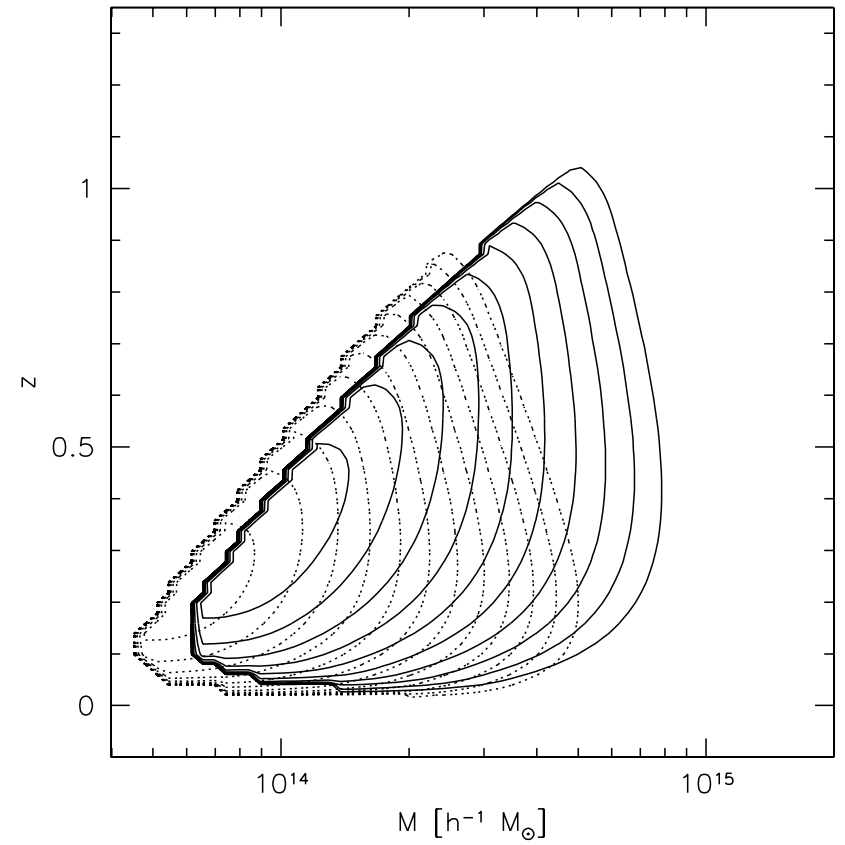

Fig. 7. Similar to Fig. 4, contours are shown of the number over all the sky of haloes in the $M-z$ plane which produce a significant aperture mass. The haloes are assumed to have NFW density profiles. The solid and dotted sets of contours refer to the $\Lambda$ CDM and SCDM models, respectively. As in Fig. 4, the contour levels range from $10^{-10.5}$ to $10^{-7} h M_{\odot}^{-1}$ and are spaced by 0.25 dex. For high $\Omega_{0}$, the contours shift to lower mass because haloes in high-density universes tend to be more compact, as described in the text.

the halo density profile. We modelled the halo population following the modification to the Press-Schechter mass function by Jenkins et al. (2001), and their density profiles as either singular isothermal or NFW profiles. As a measure for weak gravitational lensing, we chose the aperture 
mass, which is a weighted integral over the scaled surface mass density within a circular aperture. Due to its direct relation to the gravitational tidal field, the aperture mass $M_{\text {ap }}$ is a directly observable quantity. We call the expected weak lensing signal significant if its signal-to-noise ratio is five or higher.

We further assume that the dark matter has a CDM power spectrum, normalised such that the local abundance of rich galaxy clusters is reproduced.

Our results can be summarised as follows:

1. For singular isothermal spheres, aperture mass $M_{\text {ap }}$ and physical mass $M$ are related by $M_{\text {ap }} \propto M^{2 / 3}$, for NFW haloes, by $M_{\mathrm{ap}} \propto M^{1 / 3}$. This result was found earlier (Bartelmann 2001), but we repeat it here for completeness. Depending on the density profile, the aperture mass is therefore sensitive to different scalings of the physical halo mass.

2. Changes in the relation between $M_{\text {ap }}$ and $M$ imply that the physical halo mass range probed by $M_{\text {ap }}$ depends on the density profile. It is wider if the relation is flatter, hence $M_{\text {ap }}$ probes a larger physical mass range if the haloes have NFW profiles.

3. Weak-lensing measures like $M_{\text {ap }}$ are sensitive to haloes with masses $M \gtrsim 5 \times 10^{13} h^{-1} M_{\odot}$, above the nonlinear mass today, $M_{*} \sim 10^{13} h^{-1} M_{\odot}$. Therefore, in the mass range probed by weak lensing, the halo mass function steepens from the power-law to the exponential fall-off. Even moderate extensions of the mass range therefore lead to substantial changes in the detectable halo numbers.

4. The mass range probed in low-density universes $\left(\Omega_{0} \sim\right.$ $0.3)$ is $\sim(1.5 \pm 0.9) \times 10^{14} h^{-1} M_{\odot}$ for NFW haloes, and notably shifted to higher masses for singular isothermal haloes, $\sim(4.8 \pm 1.5) \times 10^{14} h^{-1} M_{\odot}$. In highdensity universes $\left(\Omega_{0} \sim 1.0\right)$, typical masses are lower; $\sim(1.0 \pm 0.5) \times 10^{14} h^{-1} M_{\odot}$ for NFW, and $\sim(2.1 \pm 0.7) \times$ $10^{14} h^{-1} M_{\odot}$ for singular isothermal haloes.

5. For low-density universes, the redshift range of the weak-lensing haloes is $\sim(0.4 \pm 0.18)$, while it is slightly narrower and shifted to somewhat lower redshift, $\sim(0.3 \pm 0.14)$, for high-density universes. The redshift range is fairly independent of the halo density profile.

6. Quite independent of the cosmological parameters, the expected number of significantly lensing haloes per square degree is $\sim 10$ if the haloes have NFW density profiles, and approximately an order of magnitude less if they are singular isothermal spheres. The somewhat surprising result that the number of weaklensing haloes does not substantially change with cosmic density is due to the balance between halo evolution and halo compactness. While the number of haloes decreases much more rapidly with increasing redshift in a high-density universe, their virial overdensity is higher, giving rise to a stronger lensing signal. As illustrated in Fig. 7, this allows the detection of haloes with somewhat smaller mass, which significantly increases the detectable halo number because of the steepness of the mass function.

These results demonstrate the possibility to constrain the density profile of dark matter haloes by counting how many haloes per square degree produce a significant weak lensing signal. Of course, dark-matter density profiles can also be directly measured using weak lensing methods. However, such measurements suffer from the intrinsic resolution limit of weak lensing due to the finite number of background galaxies, and the fact that the NFW density profile has approximately isothermal slope in the radial range where weak-lensing measures are most sensitive. Furthermore, King \& Schneider (2001) investigated how easy it is to distinguish between NFW and powerlaw profiles, using a maximum likelihood approach. It was concluded that wide field images are required to put a significant constraint on the density profile of an individual cluster.

Acknowledgements. This work was supported by the TMR Network "Gravitational Lensing: New Constraints on Cosmology and the Distribution of Dark Matter" of the EC under contract No. ERBFMRX-CT97-0172. We would like to thank Volker Springel and Simon White for very kindly allowing us to use their cluster simulations, and Houjun Mo for his careful reading of the manuscript.

\section{References}

Bacon, D., Refregier, A. \& Ellis, R. 2000, MNRAS, 318, 625

Bartelmann, M. 1996, A\&A, 313, 697

Bartelmann, M. 2001, A\&A, 370, 754

Bartelmann, M., \& Schneider, P. 2001, PhR, 340, 291

Clowe, D. I., Luppino, G. A., Kaiser, N., \& Gioia, I. M. 2000, ApJ, 539, 540

Eke, V. R., Cole, S., \& Frenk, C. S. 1996, MNRAS, 282, 263

Eke, V. R., Navarro, J. F., \& Frenk, C. S. 1998, ApJ, 503, 569

Erben, T., et al. 2000, A\&A, 355, 23

Fischer, P., \& Tyson, A. J. 1997, AJ, 114, 14

Gradshteyn, I. S., \& Ryzhik, I. M., Table of Integrals, Series, and Products, fourth edition (Academic Press, New York, San Francisco, London), 1965

Hoekstra, H., Franx, M. \& Kuijken, K. 2000, ApJ, 532, 88

Hoekstra, H., et al. 2001, ApJ, 548L, 5

Jenkins, A., Frenk, C. S., White, S. D. M., et al. 2001, MNRAS, 321,372

Kaiser, N., \& Squires, G. 1993, ApJ, 404, 441

Kaiser, N., Squires, G., Fahlman, G., \& Woods, D. 1994, in Clusters of galaxies, Proc. XIVth Moriond astrophysics meeting, Méribel, France, 269

Kaiser, N., Wilson, G., \& Luppino, G. A. 2000, ApJ, in press [astro-ph/0003338]

King, L. J., Clowe, D. I., Schneider, P., \& Springel, V. 2000, IAU Symp., 201, 67

King, L. J., \& Schneider, P. 2001, A\&A, 369, 1

Kruse, G., \& Schneider, P. 1999, MNRAS, 302, 821

Łokas, E. L., \& Hoffman, Y. 2001, in Proc. 3rd International Workshop on the Identification of Dark Matter (IDM2000), in press [astro-ph/0011295]

Maoli, R., Van Waerbeke, L., Mellier, Y., et al. 2001, A\&A, 368,766 
Mathiesen, B. F., \& Evrard, A. E. 2001, ApJ, 546, 100

Mellier, Y. 1999, NATO Science Series, Series C, Math. Phys. Sci., 541, 221

Navarro, J. F., Frenk, C. S., \& White, S. D. M. 1995, MNRAS, 275,720

Navarro, J. F., Frenk, C. S., \& White, S. D. M. 1997, ApJ, 490, 493

Press, W. H., \& Schechter, P. 1974, ApJ, 187, 425

Schneider, P. 1996, MNRAS, 283, 837

Schneider, P., Van Waerbeke, L., Jain, B., \& Kruse, G. 1998, MNRAS, 296, 873

Sheth, R. K., \& Tormen, G. 1999, MNRAS, 308, 119
Sheth, R. K., Mo, H. J., \& Tormen, G. 2001, MNRAS, 323, 1

Springel, V. 1999, Ph.D. Thesis, Munich: Ludwig-MaximiliansUniversity

Stoehr, F. 1999, High Resolution Simulations of Underdense Regions, Diploma Thesis, Munich: Technical University

Van Waerbeke, L., et al. 2000, A\&A, 358, 30

Van Waerbeke, L., et al. 2001, A\&A, submitted [astro-ph/0101511]

Wittmann, D. M., Tyson, J. A., Kirkman, D., Dell'Antonio, I., \& Bernstein, G. 2000, Nature, 405, 143

Wu, X.-P., Fang, L. Z., \& Xu, W. 1998, A\&A, 338, 813 